\title{
EFICACIA DE LA TERAPIA DE ONDAS DE CHOQUE COMO ALTERNATIVA DE TRATAMIENTO EN LESIONES DEL MANGUITO ROTADOR
}

\section{EFFECTIVENESS OF SHOCK WAVE THERAPY AS AN ALTERNATIVE TO THE ROTATOR CUFF INJURY TREATMENT}

\section{TÍTULO CORTO: EFICACIA DE LA TERAPIA DE ONDAS DE CHOQUE}

Roberto Joaquín Del Gordo-D`Amato ${ }^{1}$, Guillermo Orlando Trout-Guardiola ${ }^{2}$, José Acuña-Pinilla ${ }^{3}$

Recibido en septiembre 07 de 2015

Aceptado en octubre 26 de 2015

\section{RESUMEN}

Las lesiones del manguito rotador resultan motivo de consulta frecuente en pacientes mayores. La mayoría de las veces no existen antecedentes traumáticos agudos generando limitación progresiva en actividades la vida diaria (AVD). El objetivo de este estudio es mostrar resultados en tendinosis del manguito rotador, en pacientes tratados con terapia de ondas de choque extracorpóreas (TOCE). Se realiza un estudio observacional descriptivo prospectivo en el cual presentan resultados clínicos y funcionales en pacientes con lesión descrita, tratados con TOCE con pobre respuesta a tratamientos convencionales y cuadros clínicos de larga data mediante la aplicación de Escala Visual Análoga (EVA) del dolor y valoración del rango de movilidad. La mayor presencia de lesión se presentó en mujeres 63.6\%. El hombro mayormente afectado fue derecho en un 63.6\%. Se encontró cambios significativos en EVA pre y post tratamiento con medias de 7.9 y 0.5 respectivamente y diferencia estadística $\mathrm{p}<0.001$. Igualmente se evidenció diferencia estadísticamente significativa de los diferentes rangos de movimiento pre y post tratamiento $\mathrm{p}<0.001$. Se concluye que la TOCE es un método eficaz en el tratamiento de las tendinosis del manguito rotador con alivio del dolor y retorno a niveles funcionales.

Palabras clave: síndrome de hombro doloroso; manguito de los rotadores; tendinopatia; hombro

\section{ABSTRACT}

Rotator cuff injuries are reason for consultation frequent in elderly patients. Most of the time there are no background traumatic acute generating progressive limitations in activities of daily living (ADLS). The objective of this study is to show results in tendonitis of the rotator cuff, in patients treated with extracorporeal shock wave therapy (ESWT).

1. Ortopedista y traumatólogo, Profesor Asociado Universidad del Magdalena, Instructor AO Trauma, Miembro Asociación Colombiana de Ondas de Choque- Federación Latino - Americana de ondas de choque e International Society for Medical Shockwave Treatment. Grupo de investigación diabetes y corazón, Universidad del Magdalena, Colombia.Correo: robertoj65@hotmail.com

2. Medico Internista y Cardiólogo, Especialista en Epidemiología, Profesor Asociado Universidad del Magdalena. Grupo de investigación diabetes y corazón. Universidad del Magdalena, Colombia. Correo: troutguillermo@yahoo.com

3. Instrumentador Quirúrgico Profesional. Especialista en Epidemiología Clínica. Docente Universidad de Santander, Bogotá - Colombia. Correo: joacupi@gmail.com 
It is a prospective descriptive observational study which presents clinical and functional outcomes in patients with described lesion, treated with ESWT with poor response to conventional treatments and clinical pictures of longstanding through implementing visual analog scale (VAS) of pain and evaluation of range of motion. The greater presence of lesion is present in women $63.6 \%$. Mostly affected shoulder was right in a $63.6 \%$. Found significant changes in VAS pre and post treatment with averages of 7.9 and 0.5 respectively and different statistical p $<0.001$. We were conclude that the ESWT is an effective method in the treatment of the tendonitis of the rotator cuff with relief from pain and return to functional levels.

Keywords: Shoulder Impingement Syndrome; Rotator cuff; Tendinopathy; Shoulder

\section{INTRODUCCIÓN}

$\mathrm{L}$ as lesiones del manguito rotador resultan un motivo de consulta frecuente en diversos centros de atención, y esta lesión puede generarse en trauma agudo pero la inmensa mayoría de la veces es generada por micro traumas repetitivos en esta región anatómica ${ }^{1}$. Los pacientes de sexo femenino con edad superior a 50 años son mayormente afectados que los de sexo masculino en razón 3:1, situación explicable en razón a los cambios hormonales post menopaúsicos en este tipo de pacientes ${ }^{2}$. Del mismo modo muchas de estas lesiones se encuentran asociadas a calcificaciones del espacio sub acromial y fundamentalmente en la inserción del tendón del supra espinoso $0^{3-5}$.

Los tratamientos conservadores convencionales en lesiones del manguito rotador resultan en una tasa de eficiencia del 60 al $75 \%$ de acuerdo a diversas series publicadas y estos incluyen, antinflamatorios no esteroideos, terapia física e infiltraciones con corticoesteroides. En los fracasos del tratamiento conservador la cirugía abierta o artroscópica resultan en la otra opción de tratamiento y por lo general se indican ante el fracaso de los tratamientos conservadores $\mathrm{y}$ en rupturas o desinserciones del manguito rotador ${ }^{6-8}$.

En los últimos veinte años la terapia de ondas de choque extracorpóreas (TOCE) se han planteado como una alternativa en el tratamiento de diversas lesiones musculo esqueléticas ${ }^{9,10}$, con variabilidad de resultados en cuanto a la curación de las lesiones ${ }^{11}$, pero con eficacia comprobaba 24 en cuanto a la analgesia y mejoría funcional en diversas patologías ${ }^{1,12,13}$ tales como fascitis plantar, tendinosis del manguito rotador, eipcondilitis lateral e incluso retardos de consolidación y pseudoartrosis ${ }^{14-17}$.

No obstante, diversos estudios cuestionan la verdadera eficacia en cuanto a la aplicación de TOCE en algunos tipo de patologías musculo esqueléticas ${ }^{18,19}$.
El efecto terapéutico de las TOCE ha sido documentado en diversos estudios in vitro e in vivo mediante dos mecanismos básicos de acción. El primero de ellos corresponde a una revascularización celular por procesos de angio neo génesis, y el segundo de ellos mediante procesos biomoleculares $\mathrm{d}$ e reemplazo de fibrocitos por colágeno $0^{20-24}$.

A través del tiempo mas de ocho mil pacientes han sido tratados de diversa lesiones musculo esqueléticas en diversa publicaciones ${ }^{25} \mathrm{y}$ dentro de este grupo de pacientes un porcentaje corresponde a lesiones del manguito rotador con o sin calcificación asociada ${ }^{26-31}$.

Las tasas de curación de las lesiones del manguito rotador oscilan en diversos estudios entre el 76 y el $84 \%$ mediante la aplicación de TOCE con mejoría funcional en más del $60 \%$ de estos pacientes ${ }^{32}$.

Los efectos secundarios descritos en la literatura incluyen aumento del dolor posterior a la primera sesión, petequias y hematomas, fundamentalmente con ondas de choque de alta energía no así con las de baja energía ${ }^{12,18,33,34}$.

En lesiones no calcificadas del manguito rotador, Schmith et $a l .{ }^{35}$ recomiendan realizar el tratamiento con 6000 impulsos a dosis de $0.11 \mathrm{~mJ} / \mathrm{mm}^{2}$ en una solo sesión. Similares resultados reportan Spped et al. ${ }^{36}$, mediante la aplicación de 1500 impulsos con intervalo semanal durante tres sesiones a dosis de $0.12 \mathrm{~mJ} / \mathrm{mm}^{2}$ con hombro funcional.

De otro lado los efectos analgésicos de las TOCE en lesiones del manguito rotador han sido documentados por diversos autores con resultados mantenidos a través del tiempo de hasta 12 meses posterior a la finalización del tratamiento ${ }^{1,13,36,37}$.

El objetivo de este estudio es mostrar resultados en tendinosis del manguito rotador, en pacientes tratados con 
terapia de ondas de choque extracorpóreas mediante la evaluación del dolor pre y post tratamiento y evaluación del rango de movilidad pre y post tratamiento.

\section{MATERIALES Y MÉTODOS}

Se diseña un estudio observacional descriptivo prospectivo tipo serie de casos en el cual se incluyen todos los pacientes tratados con TOCE para lesión del manguito rotador, entre el periodo comprendido de julio 2012 a agosto de 2013. Los pacientes se atendieron y evaluaron en la institución Consultorio Médico de Ortopedia y Traumatología, diagnosticados clínica e imagenlógicamente mediante ejecución de resonancia magnética de hombro, con lesión del manguito rotador de tipo tendinosis (Figura 1). Todos los casos tenían tratamiento previo con analgésicos, anti inflamatorios no esteroideos, terapia física mínimo treinta (30) sesiones e infiltraciones en tres ocasiones con intervalo semanal, sin mejoría en síntomas mayor al 50\% de su cuadro inicial, EVA de mínimo 5/10 y con persistencia de sintomatología en un periodo mayor de seis (6) meses. Fueron excluidos del presente estudio pacientes con cirugía previa del manguito rotador y diagnóstico imagenológico de ruptura del manguito rotador.

Todos los casos fue tratados mediante la utilización de equipo de ondas de choque BTL $6000^{R}$ y según protocolo de Fenway medical mediante comunicación personal, en tendinopatias insercionales del manguito rotador, tres sesiones con intervalo semanal de 2000 disparos en cada fase, aplicando una fase de desensibilización con baja intensidad (1.5 Barr) y alta frecuencia $(12 \mathrm{~Hz})$ con una fase terapéutica con alta intensidad (2.5 Barr ) y baja frecuencia $(10 \mathrm{~Hz})$, culminando cada esquema con una última fase con aplicación de neuromodulación analgésica con 15 Barr de intensidad y $15 \mathrm{~Hz}$ de frecuencia. Ninguno de los pacientes incluidos en el presente estudio requirió de anestesia local durante la aplicación de TOCE y en todos ellos se indicó la aplicación de lidocaína tópica en parches y analgésicos vía oral (acetaminofén) durante siete días posteriores a la aplicación de la primera sesión.

Se realizó evaluación del dolor por escala análoga visual (EVA) pre tratamiento y posterior a las tres sesiones de TOCE con registros de una semana posterior a la aplicación de las ondas de choque del impacto de cada sesión y la escala funcional Simple Shoulder Test (SST) y Universidad of Pennsylvania Shoulder Score (PSS) ${ }^{39}$ entre la condición previa al tratamiento y tres semanas posterior a la culminación.
Los pacientes manifestaron su voluntad y conformidad con el desarrollo del presente estudio para la realización del tratamiento firmando un consentimiento informado conforme a lo establecido en la resolución 8430 de 1993 del ministerio de salud en lo referente a las normas éticas, técnicas y administrativas.

Los datos se recolectaron y compararon mediante pruebas no paramétricas para muestras emparejadas. Se utilizó para el análisis programa estadístico SPSS versión 20.

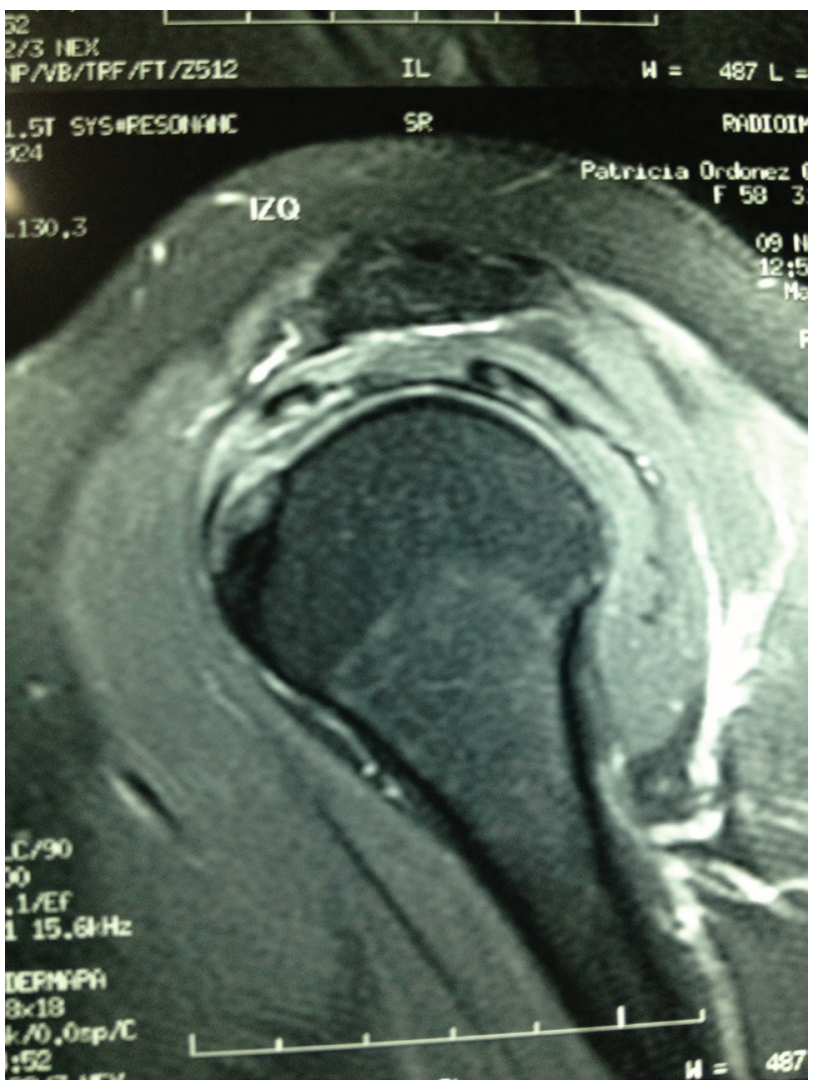

Figura 1. Imagen de resonancia magnética que muestra tendinosis del supra espinoso en el manguito rotador.

\section{RESULTADOS}

Los pacientes recibieron atención médica para tratamiento de TOCE por lesión del manguito rotador, entre el periodo de julio 2012 a agosto de 2013 representaron un total de 22 , de los cuales la mayor cantidad era del género femenino $63.6 \%$ (14), con edad media general de 62 años (D.E. 14.7). Las mujeres tenían edad media de 66 
años (D.E. 10.7) y los hombres 54 años (D.E. 18.6) sin diferencia estadística entre géneros $\mathrm{p}=0.267$. El hombro que mayor frecuencia resultó afectado, el derecho $63.6 \%$ (14) y vinculado al género femenino representa en $40.9 \%$ (9) del total.

La EVA se reportó previo al inicio del tratamiento con ondas de choque con media de 7.9 (D.E. 1.4) y una semana posterior a cada sesión del tratamiento presentando cambios en cada una de las sesiones con una media al final de 0.5 (D.E. 0.7) (Figura 2), con diferencias estadísticas significativas $\mathrm{p}<0.001$.

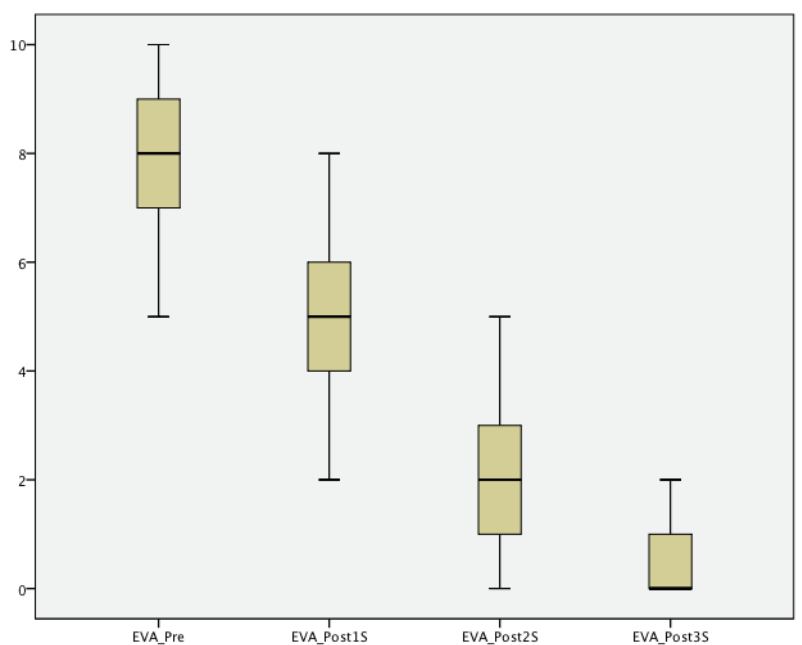

Figura 2. Escala Análoga Visual de Dolor (EVA)
Las mediciones funcionales de los arcos de movimientos flexión anterior, extensión, abducción, aducción y rotación externa, presentaron cambios entre el pre tratamiento y los logrados al final del mismo con incrementos de $15.38 \%, 41.18 \%, 25.0 \%$, $32.91 \%$ y $35.71 \%$ respectivamente, (Figura 3). Los cambios fueron estadísticamente significativos con p <0.001 (Tabla 1).

Se presentó un caso con complicación equivalente al $4.5 \%$, y se reportó como hematoma local manejado medicamente mediante la aplicación de calor local y fue superado después de 6 días y previo a la realización de la segunda sesión.

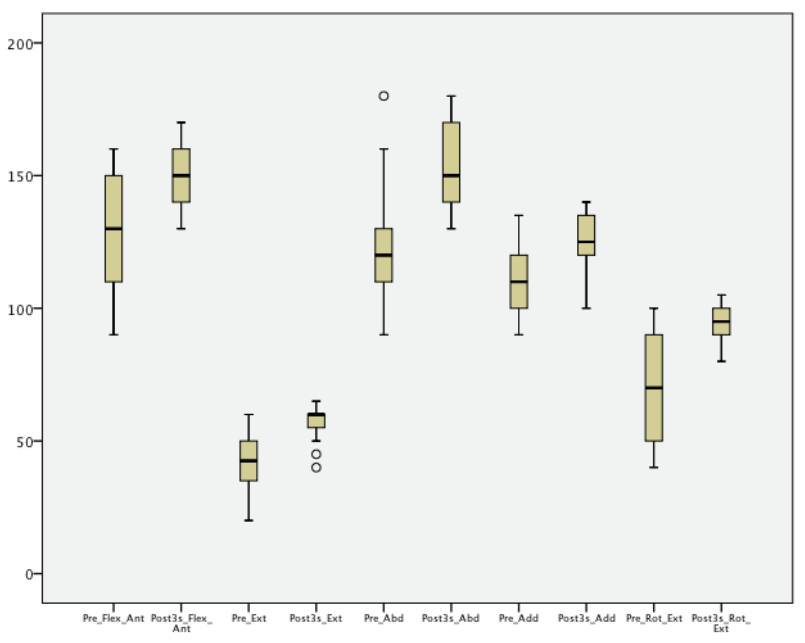

Figura 3. Arcos de Movimiento pre y post tratamiento con ondas de choque

Tabla 1. Características basales de la población de estudio

\begin{tabular}{|c|c|c|c|c|c|c|}
\hline & & $\begin{array}{c}\text { Pre } \\
\text { Tratamiento }\end{array}$ & $\begin{array}{c}\text { Post } \\
\text { 1ra Sesión }\end{array}$ & $\begin{array}{c}\text { Post } \\
\text { 2da Sesión }\end{array}$ & $\begin{array}{c}\text { Post } \\
\text { 3ra Sesión }\end{array}$ & Valor $p$ \\
\hline \multicolumn{7}{|c|}{ Escala Análoga de Dolor EVA } \\
\hline Media (DE) & & $7.9(1.4)$ & $5.1(1.5)$ & $2.2(1.3)$ & $0.5(0.7)$ & $<0.001^{*}$ \\
\hline Mediana (RIQ) & & $8.0(7.0-9.0)$ & $\begin{array}{l}5.0(4.0- \\
6.0) \\
\end{array}$ & $\begin{array}{c}2.0(1.0- \\
3.0) \\
\end{array}$ & $0.0(0.0-1.0)$ & \\
\hline \multicolumn{7}{|c|}{ Mediciones Funcionales Arcos de Movimientos } \\
\hline \multirow[b]{2}{*}{ Flexión Anterior } & Media (DE) & $129.1(21.8)$ & & & $152.1(10.9)$ & $<0.001^{*}$ \\
\hline & Mediana (RIQ) & $\begin{array}{c}130.0(107.5- \\
150.0) \\
\end{array}$ & & & $\begin{array}{c}150.0(140- \\
160.0)\end{array}$ & \\
\hline \multirow[t]{2}{*}{ Extensión } & Media (DE) & $42.9(10.9)$ & & & $56.8(6.1)$ & $<0.001^{*}$ \\
\hline & Mediana (RIQ) & $42.5(33.7-50.0)$ & & & $\begin{array}{l}60.0(53.8- \\
60.0)\end{array}$ & \\
\hline
\end{tabular}


Continuación Tabla 1.

\begin{tabular}{|c|c|c|c|c|c|c|}
\hline & & $\begin{array}{c}\text { Pre } \\
\text { Tratamiento }\end{array}$ & $\begin{array}{c}\text { Post } \\
\text { 1ra Sesión }\end{array}$ & $\begin{array}{c}\text { Post } \\
\text { 2da Sesión }\end{array}$ & $\begin{array}{c}\text { Post } \\
\text { 3ra Sesión }\end{array}$ & Valor $\mathbf{p}$ \\
\hline \multicolumn{7}{|c|}{ Mediciones Funcionales Arcos de Movimientos } \\
\hline \multirow[b]{2}{*}{ Abducción } & Media (DE) & $121.4(22.7)$ & & & $153.6(18.1)$ & $<0.001^{*}$ \\
\hline & Mediana (RIQ) & $\begin{array}{c}120.0(107.5 \text { - } \\
130.0)\end{array}$ & & & $\begin{array}{c}150.0(138.8- \\
170.0) \\
\end{array}$ & \\
\hline \multirow[b]{2}{*}{ Aducción } & Media (DE) & $110.2(11.6)$ & & & $125.0(13.2)$ & $<0.001^{*}$ \\
\hline & Mediana (RIQ) & $\begin{array}{l}110.0(100.0 \text { - } \\
120.0)\end{array}$ & & & $\begin{array}{c}125.0(117.5- \\
136.3)\end{array}$ & \\
\hline \multirow{2}{*}{ Rotación Externa } & Media (DE) & $71.4(20.9)$ & & & $94.9(6.5)$ & $<0.001^{*}$ \\
\hline & Mediana (RIQ) & $70.0(50.0-92.5)$ & & & $\begin{array}{c}95.0(90.0- \\
100.0)\end{array}$ & \\
\hline
\end{tabular}

* Diferencia estadística significativa

\section{DISCUSIÓN}

La terapia con ondas de choque extracorpóreas de baja energía resulta una alternativa de tratamiento en tendinosis y tendinitis calcificadas del manguito rotador coincidiendo con los estudios reportados por Speed et $a l .{ }^{1}$, Refior et al ${ }^{5}$ y Lohse-Busch et al. ${ }^{27}$.

En lo referente a la aplicación de EVA en tendinopatias insercionales del manguito rotador, nuestro reporte coincide con los realizados por Haake et al..$^{33}$ y Rompe et al. $^{37}$ en cuanto al alivio del dolor en lo referente a la calificación previa y post tratamiento con hallazgos estadísticamente significativos.

Del mismo modo, se encontraron resultados similares en cuanto a recuperación funcional post tratamiento con tres sesiones a intervalo semanal en los hallazgos de nuestro estudio y los reportados por otros autores tales como Harniman et al..$^{30}$ y Rompe et al. ${ }^{32}$.

A diferencia de lo reportado por Ogden et al. ${ }^{25}$, ninguno de los pacientes incluidos en este estudio presentaron complicaciones nerviosas tipo parestesias en zona de aplicación posterior al tratamiento o intolerancia al mismo por dolor generado durante la aplicación del tratamiento. Solo uno de nuestros pacientes equivalente al $4.5 \%$ presentó hematoma en zona de aplicación como efecto secundario, coincidiendo con los reportes de Schmit et al. ${ }^{35}$ en cuanto a la baja frecuencia de aparición de complicaciones.

\section{CONCLUSIÓN}

La TOCE es un método seguro y eficaz en el tratamiento de las lesiones tipo tendinosis del manguito rotador fundamentalmente del supra espinoso, con alivio del dolor y retorno a niveles funcionales en cuanto a rango de movilidad de hombro de acuerdo a los hallazgos reportados en el presente estudio.

Se recomienda realizar estudios similares que incluyan pacientes con otras tendinopatias insercionales que permitan corroborar eficacia en aplicación de ondas de choque extracorpóreas como alternativa de tratamiento, de tal forma que se eviten intervenciones quirúrgicas indicadas en forma prematura en este tipo de patologías y que representan un mayor riesgo de complicaciones en estos pacientes.

\section{DECLARACIÓN SOBRE CONFLICTO DE INTERESES}

Los autores no reportan conflictos a declarar relacionados con la investigación. 


\section{REFERENCIAS BIBLIOGRÁFICAS}

1. Speed CA, Richrards C, Nicholds D, Burnet S, Wies JT, Humphreys $\mathrm{H}$, et al. Extracorporeal shock wave therapy for tendonitis of rotator cuff: a doublé blind randomized controlled trial. J Bone Joint Surg Br. 2002; 84(4):509 - 12

2. Ortham Z, Alper M, Akman Y, Yavuz O, Yalciner A. An experimental study on the application of extracorporeal shock waves in the treatment of tendon injuries: a preliminary report. J Orthop Sci. 2001; 6 (6): 566 - 70.

3. Bosworth BM. Calcium deposits in the shoulder and subacromial bursitis: a survey of 12,122 shoulders. JAMA. 1941; (116): 2477- 82.

4. Uhthoff HK, Sarkar K. Calcifiyng tendinitis. In: Rockwood CA, Matsen FA III, eds. The Shoulder. Philadelphia, Pa: WB Saunders Co; 1990: 774 - 90.

5. Refior HJ, Krodel A, Melzer C. Examinations of the pathology of the rotator cuff. Arch Orthop Trauma Surg. 1987;106 (5): $301-8$.

6. Hollis S, Campbell $\mathrm{F}$. What is meant by intention-to-treat analysis? Survey of a published randomised controlled trials. BMJ. 1999;319 (7211): 670 - 4.

7. Ark JW, Flock TJ, Flatow EL, Bigliani LU. Arthroscopic treatment of calcific tendinitis of the shoulder. Arthroscopy. 1992;8 (2):183 - 8.

8. Uhthoff HK, Sarkar K. Calcifying tendinitis. Baillieres Clin Rheumatol. 1989; 3 (3): 567- 81.

9. Malay DS, Pressman MM, Assili A, Kline JT, York S, Buren B, et al. Extracorporeal shockwave therapy versus placebo for the treatment of chronic proximal plantar fasciitis: results of a randomized, placebo controlled, double- blinded, multicenter, intervention trial. J Foot Ankle Surg. 2006; 45 (4): 196 - 210.

10. Wess O, Ueberle F, Durhben RN. Working group technical developments: consensus report in high energy shock waves in medicine. In: Chaussy C, Eisenberger F, Jocham D, Wilbert D, eds. High-energy shock waves in medicine. Thieme Stuttgart, 1997.

11. Haake M, Konig IR, Decker T, Riedel D, Buch M, Müller HH. Extracorporeal shock wave therapy in the treatment of tennis elbow: results from a prospective randomized placebo-controlled multicenter trial. J Bone Joint Surg [Am]. 2002; 84-A (11): 1982-91.

12. Rompe JD, Hopf C, Kullmer K, Heine J, Burguer R, Nafe B. Low energy extracorporeal shock wave therapy for persistent tennis elbow. Orthop.1996; 20 (1): 23 - 7.

28 13. Green S, Buchbinder R, Glazier R, Forbes A. Systematic review of randomised controlled trials of interventions for painful shoulder: selection criteria, outcome assessment, and efficacy. BMJ. 1998; Vol 316 (7128): $354-60$.

14. Schleberger R, Senge T. Non-invasive treatment of longbone pseudarthrosis by shock waves (ESWL). Arch Orthop Trauma Surg. 1992; 111(4): 224 - 7.
15. Ogden JA, Alvarez R, Levitt R, Cross GL, Marlow M. Shock wave therapy for chronic proximal plantar fasciitis. Clin Orthop. 2001; (387): 47 - 59.

16. Cosentino R, Falsetti P, Manca S, De Stefano R, Frati E, Frediani B. Efficacy of extracorporeal shock wave treatment in calcaneal entheophytosis. Ann Rheum Dis. 2001; 60 (11): 1064 - 7.

17. Buchbinder R, Ptasznik R, Gordon J, Buchanan J, Prabaharan V, Forbes Ultrasound-guide extracorporeal shock wave therapy for plantar fasciitis: a randomized controlled trial. JAMA. 2002; 288 (11): 1364 - 72.

18. Sistermann R, Katthagen BD. Complications, side effects and contraindications in the use of medium and highenergy extracorporeal shockwaves in orthopaedics. $Z$ Orthop Ihre Grenzgeb 1998; 136 (2): 175 - 81.

19. Rompe JD, Ku llmer K, Riehle HM. Effectiveness of lowenergy extracorporeal shock waves for chronic plantar fasciitis. Foot Ankle Surg 1997;(2): 215 - 21.

20. Delius M, Draenert K, Draenert Y, Börner M. Effect of extracorporeal shock waves on bone: a review of shock wave experiments and the mechanism of action of shock wave action. In: Sibert W, Buch M, Eds. Extracorporeal shock waves in ortho- paedics. Berlin: Springer-Verlag, 91-108.

21. Ogden JA, Toth-Kischkat A, Schultheiss R. Principles of shock wave therapy. Clin Orthop 2002; (387): 8 -17.

22. Perlick L, Korth O, Wallny T, Wagner U, Hesse A, Smichtt 0 . The mechanical effects of shock waves in extracorporeal shock wave treatment of calcific tendinitis: an in vitro model. Z Orthop Ihre Grenzgeb 1999; 137 (1): $10-16$

23. Gambihler S, Delius M, Brendel W. Biological effects of shock waves: cell disrup- tion, viability, and proliferation of L1210 cells exposed to shock waves in vitro. Ultrasound Med Biol 1990; 16 (6): 587 - 94.

24. Berta L, Fazzari A, Ficco AM, Maurici P Graziella EM, Frairia R. Extracorporeal shock waves enhance normal fibroblast pro- liferation in vitro and activate mRNA expression for TGF-1 and for collagen types I and III. Acta Orthopaedica 2009; 80 (5): 612-7

25. Ogden JA, Alvarez, RG, Levitt R, Marlow M, Shock Wave Therapy in Musculoskeletal Disorders. Clin Orthop 2001; (387): 22 - 40.

26. Diesch R, Haupt G. Extracorporeal Shock Wave Treatment of Pseudarthrosis, Tendinosis Calcarea of the Shoulder and Calcaneal Spur. In Siebert W, Buch M (eds). Extracorporeal Shock Waves in Orthopae- dics. Berlin, Springer Verlag. 1997; 131-135.

27. Lohse-Busch H, Kraemer M, Reime U. The Use of Extracorporeal Shock Wave Fronts for Treatment of Muscle Dysfunction of Various Etiologies: An Overview of First Results. In Siebert W, Buch M (eds). Extracorporeal Shock Waves in Orthopae- dics. Berlin, Springer Verlag. 1997; 215 -230. 
28. Rompe JD, Burger R, Hopf C, Eysel P. Shoulder function after extracorporeal shock wave therapy for calcific tendonitis. J Shoulder Elbow Surg. 1998; 7 (5): 505 - 9.

29. Siebert W, Buch M (Eds): Extracorporeal Shock- waves in Orthopaedics. New York, Springer Verlag 1997.

30. Harniman E, Carette S, Kennedy C, Beaton D. Extracorporeal shock wave therapy for calcific and non calcific tendonitis of the rotator cuff: a systematic review. J Hand Ther 2004; 17 (2): 132 - 51.

31. Loew M, Daecke W, Kusnierczak D, Ranmauzaden M, Ewerbeck V. Shock- wave therapy is effective for chronic calcifying tendinitis of the shoulder. J Bone Joint Surg [Br] 1999; 81(5):863 - 7.

32. Rompe JD, Zoellner J, Nafe B. Shock Wave Therapy Versus Conventional Surgery in the Treatment of Calcifying Tendinitis of the Shoulder. Clin Orthop 2001; (387): 72 - 82.

33. Haake M, Boddeker IR, Decker T, Buch M, Voguel M, Labek $\mathrm{G}$ et al. Side-effects of extracorporeal shock wave therapy (ESWT) in the treatment of tennis elbow. Arch Orthop Trauma Surg 2002; 122(4): 222 - 8.

34. Rompe JD, Kirpatrick CJ, Kullmr K, Schwitalle M, Krischek O. Dose related effects of shock waves on rabbit tendon achillis: a sonographic and histological study. J Bone Joint Surg [Am] 1998; 80 (3): 546 - 52.
35. Schmitt J, Haake M, Tosch A, Hildelbrand R, Deike B, Griss P. Low-energy extracorporeal shock wave treat- ment (ESWT) for tendinitis of supraspinatus: a prospective randomised study. J Bone Joint Surg [Br] 2001; 83 (6): 873 - 6.

36. Speed CA, Hazleman BL. Shoulder pain. Clin Evi. 2002;(7): 1122 - 39.

37. Rompe JD, Hopf C, Nafe B, Burger R. Low-energy extracorporeal shock wave therapy for painful heel: a prospective controlled single-blind study. J Orthop Trauma Surg 1996; 115 (2): 75 - 9.

38. Leal C. Protocolo de aplicación de terapias de ondas de choque extracorpóreas en tendinopatias insercionales del manguito rotador. Fenway medical. Comunicación personal. Junio del 2012.

39. Namdari S, Gautam Y, David E, Sameer N, Matthew L, Ramsey M, et al. Defining functional shoulder range of motion for activities of daily living. J Shoulder Elbow Surg. 2012; 21(9): 1177 - 83.

Para citar este artículo: Del Gordo-D’Amato R, Trout-Guardiola G, Acuña-Pinilla J. Eficacia de la terapia de ondas de choque como alternativa de tratamiento en lesiones del manguito rotador. Duazary. 2016 enero; 13 (1): 23 - 29 